\title{
A CO Search for Molecular Gas in High Mass Post-Main-Sequence Nebulae
}

\author{
J.P. Phillips and A. Mampaso \\ Instituto de Astrofisica de Canarias \\ La Laguna, Tenerife, Spain \\ N. Ukita \\ Nobeyama Radio Observatory \\ Tokyo Astronomical Observatory \\ University of Tokyo, Japan
}

P.G. Williams

Physics Department, Queen Mary College,

London E1 4NS, England

High mass post-main-sequence nebulae are characterised by a set of unusual, and in certain cases extreme physical characteristics, including large outflow velocities (cf. Phillips and Mampaso, 1988a), extremely compact high emission measure cores (Phillips and Mampaso, 1988b), a high incidence of bipolar morphology (Peimbert and Torres Peimbert, 1982), and evidence for anomalously high levels of shock excited $\mathrm{H}_{2} \mathrm{~S}(1)$ emission towards both the source cores (Phillips et al 1983, 1985) and nebular peripheries (Zuckerman and Gatley, 1988). The large central star masses also predispose these sources to rapid evolution within the H-R plane (perhaps one or two orders of magnitude more rapid than for typical PN (Schonberner 1981, 1983), and the acquisition of stellar temperatures $\mathrm{T}_{*}>10^{5} \mathrm{~K}$, giving rise to correspondingly high levels of nebular excitation.

Finally, we may note that the high progenitor mass range $2 \leq M / M_{\odot} \leq 10$ not only results in anomalous abundance ratios $\mathrm{C} / \mathrm{O}, \mathrm{He} / \mathrm{H}$, and $\mathrm{N} / \mathrm{O}$ (Becker and Iben, 1980; Peimbert and Torres Peimbert, 1982), a primary identifier for such nebulae, but also leads to relatively high shell masses $\mathrm{M}_{s}$ approaching perhaps $\sim 9 \mathrm{M}_{\odot}$. The characteristics of these shells are rather ill-defined, although it seems clear that given the rapid evolution of the central stars, and the high masses sloughed off during the $\mathrm{OH} / \mathrm{IR}$ mass-loss phase, then only a relatively small proportion can as yet have been ionised (cf. Phillips and Mampaso, 1989). Similarly, if the progenitor temperatures at time of shell ejection were less than $\sim 2500 \mathrm{~K}$, then much of the shell is likely to have been molecular (Glassgold and Huggins, 1983) - a position which for most sources is unlikely to be radically altered through subsequent photo- or shock-dissociation.

That such is the case for at least certain nebulae appears to be confirmed by a high incidence of $\mathrm{H}_{2} \mathrm{~S}(1)$ emission (cf. table 1), and several detections in the lower order rotational transitions of $\mathrm{CO}$ (e.g. Huggins and Healy, 1986; Knapp and Morris, 1985) 
- although the general incidence of such emission is rather poorly established. In the following, therefore, we have sought to survey a broad sample of such sources in the $\mathrm{J}=1-0, \mathrm{~J}=2-1$, and $\mathrm{J}=3-2$ transitions of $\mathrm{CO}$, using both the Kitt Peak NRAO 12 meter antenna (November 1987, March 1989), and the 45 meter antenna of the Nobeyama Radio Observatory (April 1989). The results of this survey are summarised in table 1.
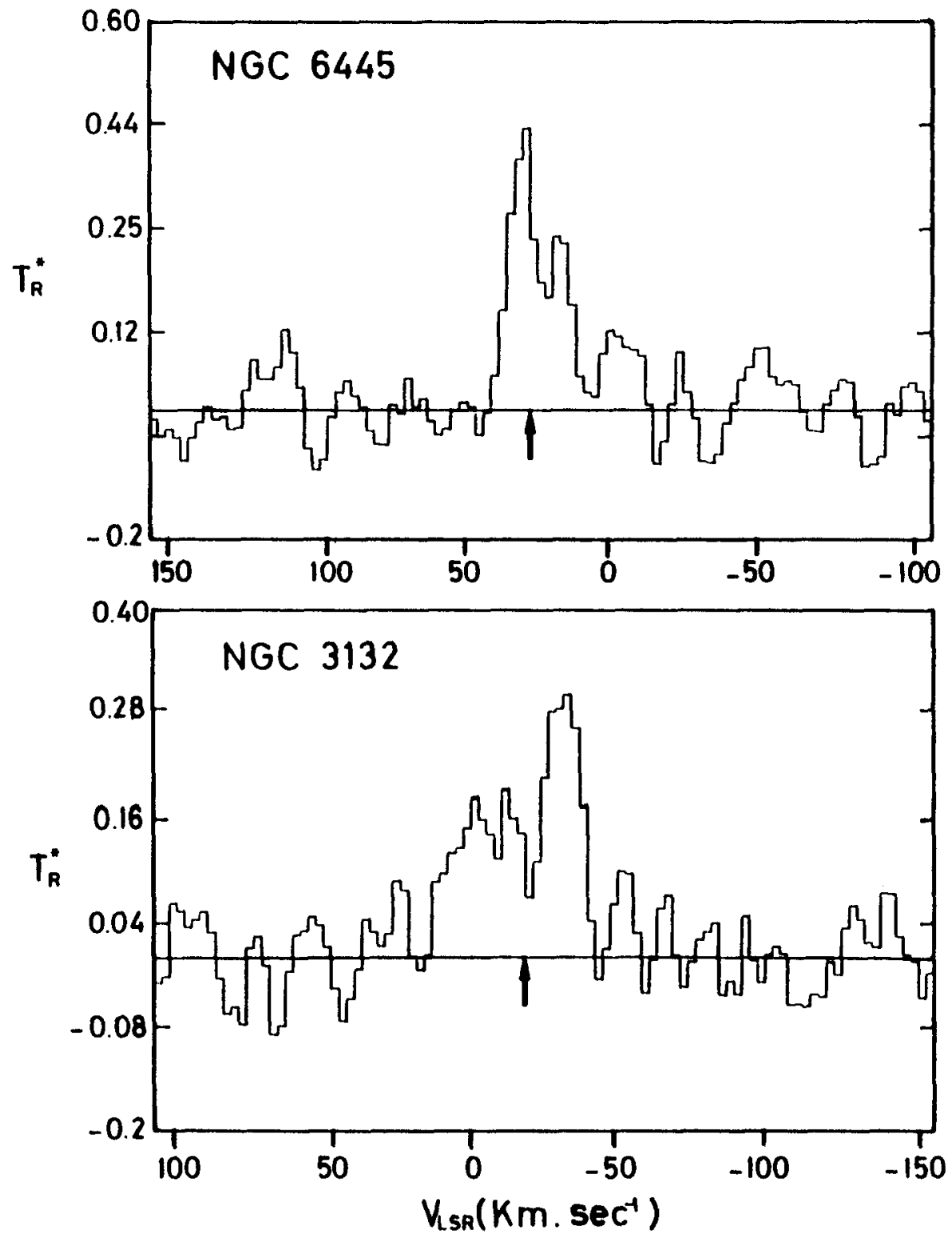

Figure 1 Two new detections of $\mathrm{CO} J=2-1$ emission in planetary nebulae, where baselines are fixed at $T_{R}^{*}=0 \mathrm{~K}$, and arrows indicate the central $\mathrm{V}_{L S R}$ of the HII regions. The results have been smoothed with a three-point Hanning function, and were in both cases acquired using the 12 metre NRAO antenna at Kitt Peak. 
It is clear, from this, that our present efforts have greatly enhanced the overall numbers of such sources measured in these transitions, although the crop of further detections appears rather meagre (we find new sources of emission in NGC 3132 and NGC 6445; see fig 1) - implying an overall detection rate of $\sim 28 \%$. This, in part, may of course arise from the rather poor limiting temperature derived for certain low latitude sources (cf. NGC 2452, NGC 6629), for which noise temperatures became prohibitively large. It is clear that several of these results would benefit from repetition at more favourable observing locations.

Nevertheless, and allowing that as many as half these lower latitude sources are eventually detected, it is clear that the overall detection limit (to $\sim 0.1 \mathrm{~K}$ ) is unlikely to exceed $\sim 36 \%$.

Such a figure is of course considerably greater than for surveys of the generality of PN - although it is perhaps still surprisingly low when considering likely shell masses. It is also of interest to notice the strong correlation between detected $\mathrm{CO}$, the incidence of $\mathrm{H}_{2} \mathrm{~S}(1)$ emission, and (less comprehensively) the presence of $21 \mathrm{~cm} \mathrm{HI}$ in either emission or absorption.

Superficially, therefore, one might conclude that yes, the high masses of such sources are indeed reflected in a high incidence of molecular emission - but that the majority of high mass shells contain little if any molecular gas.

Whether this is true in reality is less easy to discern. In particular, the absence of $\mathrm{H}_{2} \mathrm{~S}(1)$ emission in non- $\mathrm{CO}$ sources may be a partial artefact of incomplete surveys in this transition; and, although it is surely suggestive, the apparent correlation noted above may therefore be somewhat misleading. Similarly, and given that some such relationship is genuine, then this may reflect no more than an approximate correlation between $\mathrm{H}_{2} \mathrm{~S}(1)$ and $\mathrm{CO}$ emission terms; a presumption that gains some support from the similar $\mathrm{CO}$ and $\mathrm{H}_{2} \mathrm{~S}(1)$ emission distributions in certain sources (cf. Phillips and Mampaso, 1989), and evidence for related $\mathrm{CO}$ and $\mathrm{H}_{2}$ shock emission environs in nebulae such as NGC 2346 (cf. Phillips and Mampaso, 1989). Allied to similar levels of source detectability in the two transitions, then it is clear that a correlation such as observed in table 1 would be by no means surprising.

Finally, we note that reduced levels of $\mathrm{CO}$ (and $\mathrm{H}_{2}$ ) emission may arise not only from a reduced incidence of molecular gas, but also perhaps from variations in emission zone characteristics. Where $\mathrm{CO}$ emission derives primarily from a post-shock cooling zone, for instance, then high velocity gradients would lead to optically thin emission - a characteristic of at least certain of these nebulae (Phillips and Mampaso, 1989). Thus, many non-detections recorded here may simply possess rather smaller values of $\mathrm{X}(\mathrm{CO}) / \mathrm{dv} / \mathrm{dr}$, and correspondingly reduced $\mathrm{CO}$ antenna temperatures.

In short, it is clear that whilst a large fraction of such nebulae may appear to possess little molecular gas - the evidence is rather persuasive - further observations would be useful in order to assess whether this is artefact of our present, somewhat limited detection capabilities. 
Table 1

CO Survey of High Mass Nebulae

Non-Detections

$\begin{array}{lccc}\text { Source } & \mathbf{2} \sigma_{r m \boldsymbol{B}}^{1} & \mathbf{J} \rightarrow \mathbf{J}-\mathbf{1} & \text { Notes } \\ \text { NGC 650 } & 0.04 & 1-0 & 2 \\ \text { NGC 2440 } & 0.07 & 1-0 & 2,3 \\ \text { NGC 2452 } & 0.40 & 3-2 & 2 \\ \text { NGC 2818 } & 0.14 & 1-0 & 2 \\ \text { NGC 6532 } & 0.20 & 2-1 & 2 \\ \text { NGC 6629 } & 0.36 & 2-1 & 2 \\ \text { NGC 6741 } & 0.17 & 2-1 & 2 \\ \text { NGC 6751 } & 0.22 & 2-1 & 2 \\ \text { NGC 6778 } & 0.05 & 1-0 & 2 \\ \text { NGC 6853 } & 0.11 & 2-1 & 2 \\ \text { NGC 6894 } & 0.32 & 3-2 & 2 \\ \text { NGC 7008 } & 0.10 & 2-1 & 2 \\ \text { HB 5 } & ? & 2-1 & 2,6 \\ \text { Hu 1-2 } & 0.12 & 2-1 & 2,3 \\ \text { M 2-55 } & 0.06 & 1-0 & 2 \\ \text { M 3-3 } & 0.12 & 2-1 & 2 \\ \text { Me 2-2 } & 0.09 & 1-0 & 2 \\ \text { VV 47 } & 0.06 & 1-0 & 2\end{array}$

Detections

$\begin{array}{llcc}\text { Source } & \mathbf{T}_{\boldsymbol{R}}^{*} & \mathbf{J} \rightarrow \mathbf{J}-\mathbf{1} & \text { Notes } \\ \text { NGC 2346 } & 0.4 & 2-1 & 2,3,4 \\ \text { NGC 3132 } & 0.3 & 2-1 & 2,4 \\ \text { NGC 6302 } & 0.4 & 2-1 & 2,3,4,5 \\ \text { NGC 6445 } & 0.45 & 2-1 & 2 \\ \text { NGC 7027 } & 3.2 & 3-2 & 2,3,4,7 \\ \text { CRL 618 } & 1.5 & 3-2 & 2,3,4,7 \\ \text { M 2-9 } & 0.1 & 2-1 & 3,4\end{array}$

Notes: 1) Results correspond to $2 \sigma_{r m s}$ upper limits; 2) Source measured in this survey; 3) Source measured previously; 4) Associated with $\mathrm{H}_{2} \mathrm{~S}(1)$ emission; 5) HI $21 \mathrm{~cm}$ emission measured in absorption/emission; 6) Source may be strongly interacting with enveloping region - emission is present at $V_{L S R}$ of the HII region, but association with source is unclear; 7 ) Results based on recent $\mathbf{J}=3-2$ CO observations by the authors at Kitt Peak. 
In either case, it is clear that most detected PN fall within our present subset of high mass PN, and a goodly fraction of such sources reveal a penchant for strong $\mathrm{CO}$ emission; although the characteristics of such emission zones remain woefully illdetermined, and further observations (in other transitions) would prove of considerable interest and utility.

\section{References}

Becker, S.A., and Iben, I., 1980. Astrophys. J. 237, 111

Glassgold, A.E., and Huggins, P.J., 1983. Mon. Not. Roy. Astron. Soc. 203, 517

Huggins, P.J., and Healy, A.P., 1986. Astrophys. J. 305, L29

Knapp, G.R., and Morris, M., 1985. Astrophys. J. 292, 640

Phillips, J.P., and Mampaso, A., 1988b. Astron. Astrophys. 190, 237

Phillips, J.P., and Mampaso, A., 1988a. in IAU Symposium No. 131, ed S. Torres Peimbert, D. Reidel Publishing Co., Dordrecht, Holland

Phillips, J.P., Reay, N.K., and White, G.J., 1983. Mon. Not. Roy. Astron. Soc. 203, 977

Phillips, J.P., White, G.J., and Harten, R., 1985. Astron. Astrophys. 145, 118

Schonberner, D., 1981. Astron. Astrophys. 103, 19

Schonberner, D., 1983. Astron. Astrophys. 272, 708

Zuckerman, B., and Gatley, I., 1988. Astrophys. J. 324, 501 